\title{
Significado de alteraciones en la salud de niños en situación de calle
}

\author{
Meaning of Health Alterations in \\ Children Living in the Streets ${ }^{1}$
}

\section{Tatiana Marcela Aristizábal Monsalve ${ }^{1}$ Álvaro Giraldo Pineda² (1) Constanza Forero Pulido 3 (D)}

\author{
1'Autor correspondente. Universidad de Antioquia (Medellín). Colombia. tatiana.aristizabal@udea.edu.co \\ 2-3Universidad de Antioquia (Medellín).Colombia. alvaro.giraldo@udea.edu.co, constanza.forero@udea.edu.co
}

\begin{abstract}
RESUMEN | INTRODUCCIÓN: Investigación realizada en la ciudad de Medellín, Colombia. OBJETIVO: Comprender el significado que tienen para los niños en situación de calle las alteraciones en la salud y cómo las tratan. METOdologíA: Estudio cualitativo, etnográfico. Se aplicaron 30 entrevistas a niños en situación de calle y se llevó diario de campo. RESULTADOS: Las alteraciones en la salud las clasifican en enfermedades causadas por Dios y enfermedades o heridas causadas por el hombre; las primeras no pueden prevenirse, las segundas sí. Las causadas por el hombre pueden ser: graves, si están relacionadas con la posibilidad de morir y requieren atención hospitalaria; serias cuando comprometen su libertad requieren ayuda y leves cuando no comprometen su independencia y las solucionan mediante la automedicación o tratamientos culturalmente determinados. Las alteraciones en la salud significan experiencia y las heridas significan venganza, valentía o vergüenza. CONCLUSIóN: Los niños en situación de calle construyen el significado de alteración de la salud, a partir de sus experiencias, en relación con la muerte y la libertad.
\end{abstract}

PALABRAS CLAVES: Enfermedad. Heridas y lesiones. Autocuidado. Antropología Cultural. Niños sin hogar

\begin{abstract}
INTRODUCTION: The research was conducted in Medellín, Colombia. OBJECTIVE: The work sought to understand the meaning of diseases and their treatment for homeless children. METHODOLOGY: Ethnographic and qualitative research. Thirty interviews were carried out to homeless children and a field diary was kept. RESULTS: Diseases were classified as those caused by God and diseases or injuries caused by men; the latter can be prevented, but the first one cannot. Diseases caused by men can be severe, if they pose the risk of death and require hospital treatment; serious, when they jeopardize freedom; and mild, when they do not place at risk their independence and are treated through self-medication or culturally determined treatment. Diseases mean experience and injuries mean revenge, courage, and shame. CONCLUSION: Homeless children build the meaning of diseases from their experience, in relation to death and freedom.
\end{abstract}

KEYWORDS: Disease, wounds and injuries. Selfcare. Cultural anthropology. Homeless children

${ }^{1}$ Este trabajo es el resultado del resumen ampliado presentado, evaluado y aceptado en el IX Congreso Iberoamericano de Investigación Cualitativa (CIAIQ2020), que tuvo lugar del 14 al 17 de julio de 2020, en la ciudad de A Coruña (Galicia). 


\section{Introducción}

En este trabajo se parte del concepto de niños que presenta la UNICEF (2019) que los considera hasta la edad de 18 años e incluye tanto a las niñas como a los niños. Los niños en situación de calle son aquellos menores de 18 años que se apropian de los espacios de la calle y la construyen socialmente para lograr sobrevivir en ella y formar su familia.

Las situaciones por las cuales atraviesan debido a las condiciones de vida que implican buscar el sustento diario no tener lugares en donde puedan dormir y descansar; estar sometidos a los cambios de temperatura y a las lluvias; no tener una forma de protegerse; consumir alimentos en mal estado; obtener el dinero por medio de la venta de su cuerpo; ingerir sustancias psicoactivas en forma excesiva y estar en contacto con diferentes personas, los exponen a tener accidentes o sufrir traumatismos y enfermedades como las de transmisión sexual, gastrointestinales, neurológicas y respiratorias, Instituto Colombiano de Bienestar Familiar (2014).

La revisión de trabajos sobre el acceso a los servicios de salud y los significados que los habitantes de calle les dan a la salud y la enfermedad encontró que la gran mayoría de ellos se refieren a la población en general, pero hay pocos que se enfoquen específicamente en los niños en situación de calle.

Con respecto a las alteraciones en la salud, Akman et al. (1999) plantean como principales motivos de consulta en la ciudad de Buenos Aires, intoxicaciones, traumatismos, afecciones dermatológicas, enfermedades de transmisión sexual y respiratorias; Pasamonik. (2009) identifica los problemas de salud que presentan los niños que asisten a un centro integral de niñez y adolescencia, primero las enfermedades de la piel, el embarazo, parto y puerperio, las enfermedades infecciosas y por último el trauma. Además de lo anterior, Forselledo (2001) plantea como consecuencia de las enfermedades el retraso en el crecimiento y las alteraciones mentales, consecuencia del estrés que se asocia a carencias económicas. Giraldo, Forero y Aristizábal (2020) identificaron que los niños en situación de calle reciben y ejercen la violencia y como consecuencia sufren lesiones; por otro lado, se considera que las enfermedades y las heridas de los niños en situación de calle son un problema de salud pública relevante.
En este trabajo se presentan las experiencias y significados que los niños en situación de calle les dan a la enfermedad y a las heridas, situaciones a las que denominan alteraciones en la salud.

Por lo tanto, este trabajo se propuso comprender el significado que tienen para los niños en situación de calle las alteraciones en la salud y cómo las tratan. Para dar respuesta al objetivo se utilizó la investigación cualitativa con enfoque etnográfico, con participación de los niños en situación de calle, lo que permitió una mayor vinculación al proceso y aseguró un mayor rigor en la investigación.

\section{Relevancia Social y Científica}

Debido a las condiciones sociales, políticas y económicas por las que atraviesa el país, esta población aumenta. Este grupo se considera vulnerable, especialmente en aspectos de salud por las enfermedades y las heridas que reciben. La mirada desde lo cultural nos permite acercarnos al conocimiento de cómo manejan ciertas enfermedades y heridas, lo que permite aprender de ellos y en el momento que se vayan a atender tener en cuenta su mirada. Por lo tanto, este estudio pretende lograr la comprensión alrededor de las alteraciones en la salud y de esta manera brindar elementos que faciliten a las instituciones y a los entes gubernamentales que trabajan con esta población, el acercamiento a los niños y que sirvan para la construcción de programas y políticas en pro de mejorar su salud.

\section{Metodología}

Dado que el trabajo pretendía conocer las experiencias de los niños en situación de calle, en lo que se refiere a las enfermedades y heridas que sufren en su cotidianidad y tratar de comprender los significados de esas experiencias, se hizo necesaria la selección de un tipo de estudio que permitiera la comprensión; que fuera flexible; que proveyera una mirada amplia y total del fenómeno; que no juzgara los pensamientos, ni los comportamientos de los participantes y finalmente que trabajara con datos no cuantificables. Por tales razones, se seleccionó como tipo de estudio la investigación cualitativa. 
Una vez definido el tipo de estudio era necesario seleccionar un enfoque que respondiera a las necesidades específicas de la investigación, como: comprender los significados que sustentan los comportamientos de los niños en situación de calle; tener conciencia de que la presencia de los investigadores en el espacio de estas personas provocaba reacciones en ellas; captar el sentido que los niños dan a sus actos y finalmente lograr el acercamiento a su cultura, representada en sus experiencias y significados, desde su mirada (mirada emic) y se optó por el enfoque etnográfico y, se adoptó el concepto de cultura dado por Clifford Geertz, que la entiende como " un conjunto de significaciones construidas socialmente" (Geertz, 2003).

\section{Técnicas}

A los participantes en el estudio, se les aplicaron treinta (30) entrevistas, cuyas preguntas estaban encaminadas a conocer las experiencias en relación con las alteraciones en la salud expresadas en sus comportamientos y los significados dados, tanto a esas experiencias, como a las enfermedades y a las heridas. En la aplicación de las entrevistas, previa solicitud de aceptación de los participantes intervinieron dos entrevistadores, en donde uno de ellos realizaba la entrevista, y cuando la daba por terminada, el segundo entrevistador le hacía preguntas al participante que el primero había omitido y aquellas que podían explicar algunas situaciones relatadas por el entrevistado o profundizar aspectos valiosos para la investigación. Posteriormente, el segundo entrevistador le hacía aportes al primero en aspectos que podían ser mejorados o ser tenidos en cuenta en próximas entrevistas.

Cada investigador llevó un diario de campo en el cual describió sus percepciones, sus temores, sus angustias, los encuentros con las personas, lo observado, las reflexiones metodológicas acerca del proceso investigativo y las actividades llevadas a cabo. Estas anotaciones fueron útiles en el proceso de análisis de la información.

\section{Población}

Los participantes en el estudio fueron niños con experiencia de vida en la calle, que habitan en el centro de la ciudad de Medellín, Colombia y con quienes se ha trabajado desde hace varios años. Niños que hubieran sufrido durante su estancia en la calle, algún tipo de enfermedad o herida y que de manera voluntaria accedieron a participar en la investigación. El trabajo contó con el apoyo de la línea de investigación Cultura y Salud, en la cual se encuentran vinculadas personas con experiencia de vida en la calle, quienes realizaron acompañamiento en algunos de los recorridos, por medio de los cuales se logró conocer otros niños, con quienes se realizaron los primeros contactos, se les explicó de qué se trataba la investigación y se les invitó a participar de ella.

\section{Análisis de la información}

Cabe anotar que el grupo de investigación incorpora representantes de la comunidad estudiada y les provee elementos de investigación, especialmente en lo relacionado con las técnicas de recolección de información y el análisis, para que puedan participar activamente. En este trabajo, al igual que en anteriores, se vincularon niños en situación de calle al proceso de análisis y se pudo constatar que tienen gran sensibilidad para identificar los códigos y construir las categorías pertinentes, además de que corrigen la mirada etic de los investigadores en relación con la información obtenida.

En el proceso de análisis se siguieron los siguientes pasos: se transcribieron las entrevistas, teniendo el cuidado de respetar el lenguaje usado por los participantes, luego se hizo una lectura individual de cada una de ellas, en la que los investigadores escribieron comentarios generales, que compartieron posteriormente en la reunión grupal en la cual se hizo una nueva lectura de la entrevista y en ella se identificaron posibles códigos, para luego, en reuniones posteriores construir categorías, además se elaboraron mapas, que ayudaron a la visualización de las categorías y a la escritura del artículo. 


\section{Criterios de rigor}

Según Castillo y Vásquez (2003), los criterios de rigor en investigación cualitativa son: la credibilidad, la auditabilidad o confirmabilidad y la transferibilidad o aplicabilidad, así como la validez y confiabilidad. Cada uno de estos criterios fueron tenidos en cuenta en la investigación. Para el logro de la credibilidad, se incluyeron participantes en el grupo de investigación, con quienes se estableció un contacto previo durante varios meses con el fin de asegurar que la información que proporcionaran fuera verídica, además los resultados fueron discutidos con los niños, quienes dieron el aval para que la información pudiera ser presentada a la comunidad por medios hablados y escritos. Para asegurar la auditabilidad o confirmabilidad, se tuvo especial cuidado en realizar una transcripción fiel de las entrevistas, así como conservar las grabaciones. La aplicabilidad queda a decisión de quienes lean la investigación, de si es posible aplicarla o no. Para lograr la confiabilidad y validez, se buscó que hubiera congruencia entre la pregunta de investigación y los elementos usados en el método, así como que los participantes fueran representativos del grupo de niños en situación de calle y que hubiera saturación de las categorías seleccionadas.

\section{Aspectos éticos}

Se tuvo en cuenta el principio de respeto, como fuente de reconocimiento a la autonomía de las personas participantes; en el transcurso de la investigación no se ofreció ningún tipo de remuneración y las entrevistas se realizaron en los lugares y horarios en que los niños se sintieron más cómodos. Se les informó la intención del estudio y se dejó claro que se podrían retirar en el momento que ellos así lo decidieran. En la socialización de los hallazgos, la información se manejó con criterios de confidencialidad; no se revelaron datos personales, para garantizar el anonimato de los participantes.

Este estudio se ajustó a las normas internacionales sobre ética en la investigación humana. Se tomaron en cuenta los aspectos establecidos en la Resolución 008430 de 1993 del Ministerio de Salud de Colombia; según el artículo 11, la investigación está clasificada como de bajo riesgo (1993) y los investigadores no tienen conflicto de intereses con el tema.

\section{Contexto}

En la ciudad de Medellín los niños recorren las calles en pequeños grupos; han abandonado su hogar para iniciar su vida en la calle, en la cual se organizan en "parches" en los que comparten y se apoyan mutuamente. Convierten la calle en su hogar, en donde viven situaciones tales como las dificultades económicas, la violencia, el "caciqueo" (acciones de dominio y agresión contra ellos), el abuso sexual, el inicio de consumo de sustancias psicoactivas y el rechazo de las personas que frecuentan la calle, como los transeúntes, los venteros ambulantes y estacionarios y los organismos de control como la policía.

Uno de los primeros sectores a donde los niños llegan es el centro de la ciudad, dadas las condiciones de este, ya que cuenta con hoteles y residencias donde pueden "hacer ratos" (vender su cuerpo) y dormir; lugares para conseguir la comida; camellar; consumir drogas; divertirse y satisfacer sus necesidades, como calmar el hambre, bañarse, resguardarse del frío y del calor. En la ciudad adecúan ciertos espacios que los identifican como propios, uno de los más frecuentados es el viaducto del metro, donde se dedican al rebusque $o$ al consumo de drogas. En esta zona también se sitúan toda clase de vendedores ambulantes, que ofrecen artículos de segunda como herramientas, electrodomésticos, ropa, zapatos, muñecos, celulares, revistas y libros viejos. Así mismo puede verse a vendedores de frutas y verduras en las carretillas o los que venden tinto y café en coches de bebés que han acondicionado para tal fin. Igualmente, en las puertas de los hoteles, se ven las niñas y adolescentes a la espera de clientes para vender sus cuerpos; generalmente son vigiladas de cerca por aquellos que a cambio de protección reciben una parte de lo que los clientes les pagan.

Todas estas vivencias en la calle hacen que se vean expuestos a los problemas y las riñas con otros, además las condiciones en las que viven les alteran la salud y les generan enfermedades contagiosas por no tener cuidado de su cuerpo. 
gonorrea.

Las alteraciones en la salud son experiencias comunes en todas las personas. Tradicionalmente la Medicina y en especial la Epidemiología, las han clasificado en enfermedades crónicas y agudas, según el tiempo de duración y en graves y leves, según los daños que causen en el organismo y los órganos que afecten. Las heridas según el compromiso de la piel y otras estructuras son denominadas simples, complejas, penetrantes y no penetrantes.

Los niños en situación de calle viven experiencias propias del ambiente, dentro de las cuales están las alteraciones en la salud, que pueden ser físicas o mentales.

La salud, física sería por ejemplo si yo me estoy sintiendo bien. Mental, ya es ¿qué pienso yo?, si pienso cosas malas, o cosas positivas.

Las alteraciones en la salud las clasifican en dos formas que tienen que ver con la concepción que tengan de ellas, según las experiencias vividas: las causadas por Dios (Ser Supremo) y las causadas por los hombres.

\section{Eso sí mi Diosito, cosas de mi Diosito}

Las primeras no tienen curación y no se pueden prevenir, como es el caso del cáncer, además son consideradas graves. Estas enfermedades no causan el rechazo de los compañeros de la calle o "parceros", con quienes conviven a diario.

Son cosas de Dios, eso es naturaleza, porque todo mundo tenemos el cáncer, sino que a unos se les desarrolla más ligero que a otros

Según los niños las alteraciones en la salud "causadas por el hombre" son las heridas de cualquier tipo y enfermedades como las de transmisión sexual adquiridas por tener relaciones con personas infectadas y que pueden ser los mismos "parceros" o "parceras" o por el contacto con los clientes en el caso de las niñas que venden su cuerpo.
También las enfermedades producidas por carecer de comida o comer demasiados dulces; estar en contacto con comidas descompuestas, elementos contaminados o personas enfermas y por la suspensión intempestiva de sustancias psicoactivas o su consumo en exceso.

Me daban muchos ataques por sobredosis, porque tiraba mucho perico (cocaína)

Los niños incluyen dentro de la concepción de heridas, los golpes que pueden recibir de sus parceros y los accidentes de tránsito (ser atropellados por un auto), así no les rompan la piel, ni les generen pérdida de sangre, las puñaladas y puntazos causados por los mismos niños en situación de calle o por "culebras" (personas a quienes se les debe algo o deben algo), los golpes ocasionados por las riñas o peleas entre los compañeros del parche (parceros).

Esa pelaita (niña) era muy asada (muy agresiva), y tuvimos una riña y me dio una puñalada que casi me mata

También reciben golpes de los transeúntes o cualquiera otra persona y de la policía que los domina o "caciquea" y les obliga a hacer ciertas cosas, de forma abusiva, generalmente de tipo sexual, a las que ellos y ellas se niegan a realizar y por eso reciben castigos representados en agresiones físicas o en

Me estaban era dando muy duro, sentía como esa tristeza de saber que ellos (los policías), me iban a dejar moreteada, aporriada (aporreada) y que iba a quedar con el dolor.

Los niños clasifican las alteraciones en la salud ocasionadas por el hombre en graves, serias y leves. Para llegar a esta clasificación usan criterios que involucran actividades propias de su forma de vida y que en cierta medida, difieren de los usados por la Medicina y la Epidemiología.

Enfermedades, por estar en la calle, los hábitos de sobrevivir siempre van llevando el riesgo por delante encierros en las estaciones de policía. 
Las enfermedades consideradas graves son aquellas que requieren atención inmediata en una institución de salud y si no se atienden pueden causar la muerte. Entre ellas están las convulsiones atribuidas al consumo incontrolado y excesivo de sustancias psicoactivas, como la cocaína y la heroína y las enfermedades de transmisión sexual en fase avanzada o el sida.

Le daban muchos ataques por sobredosis, porque tiraba mucho perico, tocaba coger con ella para el hospital.

Que le digan es que tiene sida, va a morir por sida

Las heridas son graves cuando comprometen los órganos o son muy grandes y profundas y también requieren la atención en una institución de salud.

Uno cuando coge pal hospital tiene que ser algo muy grave y se esté vaciando uno o tenga una puñalada muy grande y una herida muy grande, pues algo mayor para uno caer al hospital.

En las alteraciones graves, los niños utilizan como último recurso el hospital, al que consideran como el lugar al cual se va a salvarse o a morir. Son pocas las veces en que los niños acuden por su cuenta al hospital, la mayoría son llevados por sus parceros o por el personal de instituciones de protección.

\section{Sentía que se me iba a parar el corazón y la respiración y yo: ¿cómo?: pal hospital.}

Para muchos de los niños ir a los hospitales implica tener que soportar que los traten mal, los discriminen, o en el caso de ser heridos, que, al manipularles las heridas, se las infecten.

\section{Alteraciones serias}

Son las que les alteran su estado de ánimo y no les permiten realizar diversas actividades del diario vivir, como lo son caminar, correr, trabajar ("camellar"), comer, robar, divertirse, compartir con sus parceros, entre otras.
Esta privación de la libertad de movimiento se relaciona con una herida que no causa daños internos y que puede ser o no penetrante, pero les produce mucho dolor; también con el consumo de sustancias psicoactivas, que les generan malestar; una enfermedad de transmisión sexual en su fase inicial; el consumo abundante de dulces, que les ocasiona dolor intenso de cabeza y estómago; el no consumo de suficientes alimentos, que según ellos les produce "anemia por desnutrición"; la suspensión de la droga, que les genera padecimientos emocionales, depresiones y fatiga; además las infecciones respiratorias y gastrointestinales, que no les permiten moverse.

Hay veces me dan dolores aquí en el estómago, o cuando me siento indispuesto, me da escalofrío, pues no me puedo levantar.

Frente a las limitaciones de movimiento que les impiden realizar sus actividades diarias, los niños en situación de calle buscan la ayuda del farmaceuta, del médico o de las entidades de apoyo, para recibir tratamiento y recuperar la libertad; también consiguen dinero para acceder a consulta en las instituciones de salud, sin embargo, el acceso se ve limitado, porque requieren presentar determinados documentos que generalmente no poseen. También por el maltrato que reciben, a veces prefieren no asistir a dichos servicios.

Prefiero morirme debajo de los puentes, que ir a hacer una tramitología para un tratamiento.

\section{Alteraciones leves}

Los niños en situación de calle denominan alteraciones leves a aquellas en las cuales pueden continuar realizando una vida diaria con normalidad, cuando las actividades y la cotidianidad no se ven interrumpidas poralguna discapacidad o indisposición que imposibilite el movimiento. Las enfermedades leves, entonces, son todos los procesos infecciosos como gripas, dolores no severos y las enfermedades gastrointestinales en su fase inicial. 
El manejo que los niños en situación de calle le dan a la enfermedad leve son el autocuidado y el descanso en su lugar de vivienda, hasta que el malestar pase y se sientan en una total normalidad, no acuden a una unidad hospitalaria, ya que las enfermedades en esta fase no requieren de apoyo adicional, solo cuidado personal.

Quedarse ahí en la pieza ahí quieta y ya se está curando

Yo de gripa y cosas así, con una pastillita uno trataba de auto medicarse

Los niños en situación de calle definen heridas leves, aquellas que no son penetrantes y no afectan ningún órgano de manera grave y que son llamadas puntazos; que no requieren de asistencia médica, solo de cuidado personal, para tener un proceso de cicatrización adecuado y evitar que se infecten, para ello utilizan remedios caseros que les ofrecen sus parceros o compañeros de calle que ya han pasado por estos mismos procesos y son una buena práctica para mantener las heridas en buen estado de desinfección y cicatrización. Dentro de estas prácticas están el uso de la ceniza de cigarrillo o marihuana, el agua oxigenada y una buena desinfección con jabón rey,

Todas las noches me echaba limón con jabón rey y me refregaba bien, hasta que eso se me sanó

Cuando sufren alguna herida prefieren cubrirla para evitar las miradas y el juicio de sus compañeros o parceros.

Me limpio, me echo alcohol, agua, yo no me dejo volver a ver eso.

Los niños refieren no ir a los hospitales por dos razones, la primera es que no son heridas que les puedan causar la muerte o les coarten la libertad y prefieren tratarlas ellos mismos; y la segunda es que en los hospitales son interrogados, no son bien recibidos y refieren que las suturas realizadas allí quedan "feas" y les pueden generar infecciones.

Vieran como le quedó ese cuero, como todo recogido. Increíble, la desfiguraron, la dejaron muy mal, y eso sucede mucho con los niños de la calle.
En el tratamiento de las alteraciones leves de la salud, enfermedades y heridas lo fundamental para los niños en situación de calle es el cuidado por parte de ellos mismos o sus compañeros más cercanos y el no uso de las unidades hospitalarias.

Uno mismo se medica en la calle, se automedica

\section{Sentimientos}

Los niños se sienten estigmatizados y rechazados por sus parceros o compañeros al contraer enfermedades como el sida, porque esta enfermedad se contagia fácilmente y les causa la muerte y no tiene curación. Las formas de estigmatización están representadas en el rechazo, no buscar la compañía de los afectados por el sida, no compartir la comida, no acercarse para no ser tocados por ellos y no acceder a tener relaciones sexuales.

Es más, por el estigma que crea la enfermedad en sí, del sida

Los niños en situación de calle expresan miedo a contraer enfermedades de transmisión sexual debido a que estas les limitan las interacciones con los otros, les causan dolores y se pueden convertir en enfermedades graves.

A eso si le he tenido mucho miedo, a las enfermedades de transmisión sexual

Las heridas graves y serias les producen a los niños rabia, porque ven que su cuerpo no vuelve a ser el mismo.

Llena de rabia porque yo tuve problemas con unas lesbianas y entoes (entonces) me pegaron con un alambre de esos y me sacaron sangre

\section{Significados}

Para los niños en situación de calle las alteraciones en la salud les significan experiencia. Las experiencias les hacen tomar medidas de prevención en un futuro, como evitar el mal manejo en el consumo de sustancias psicoactivas o de alcohol y el contacto con personas que les signifiquen riesgo.

Desde eso yo experimenté y cogí la experiencia 
Como las heridas, les han marcado su cuerpo y su espíritu aprenden a defenderse y a no volver a pasar por el sitio en donde los han herido.

Uno aprende que por ahí no puede caminar a tales horas.

La experiencia les hace evitar las "culebras" y ubicarse en sitios en donde se sientan más seguros, no pelear con los otros habitantes de calle ni con quienes comparten el centro: venteros ambulantes, policía, convivir (cooperativas de vigilancia y seguridad privadas creadas según decreto ley 356 de 1994) y mantener con ellos diálogos y relaciones interpersonales adecuadas.

Convivires. son algo así como la policía, pero más violentos

Las heridas graves y serias les significan venganza, que los hace querer enfrentarse a las personas que los hirieron y cobrar esa deuda.

Queda ya con esa venganza y entonces te la voy a pegar en cualquier momento, porque usted quedó con esa venganza

Las cicatrices significan valentía y motivo de orgullo, cuando se las hacen en cualquier parte del cuerpo diferente a la cara.

Una puñalada que me pegaron peliando (peleando) me la dejó así, chévere porque esa es una reseña que lleva

uno

Las heridas son motivo de molestia y vergüenza cuando se las hacen en el rostro y por ello en las peleas, la parte del cuerpo que más protegen es este.

Yo no consiento ni que me hagan así, ahí no, la cara mía es lo mejor, pa' mi es lo mejor, porque el cuerpo a mí no, que me metan una puñalada... en el cuerpo... en

la cara no.

\section{Prevención}

La prevención la establecen según las experiencias y los significados construidos en la calle.

Los participantes tienen claro que para evitar las enfermedades causadas por el hombre deben realizar acciones como cuidar el cuerpo, lo que implica comer bien, hacer ejercicio y mantenerse aseados.
Yo hago mucho ejercicio, me lo como todo, ensaladas, eso lo ayuda a mantener una buena salud.

También recomiendan mantener la distancia con las personas que tienen alguna infección controlar la ingesta de comida y el consumo de sustancias psicoactivas.

¿Qué hace para cuidarse?, alejarse de esas personas

Una forma de prevenir el contagio de enfermedades de transmisión sexual es el uso del condón y detectarlas a tiempo (diagnosticarlas), antes de tener la relación sexual y para ello aplican diferentes técnicas, que consideran efectivas, en las cuales emplean elementos como la ceniza de la marihuana, el zumo o jugo de limón y la cerveza y si hay reacción concluyen que la persona está infectada y evitan la relación.

En el caso de las mujeres se les aplica limón en la vagina y si manifiestan alguna incomodidad se concluye que tienen una enfermedad de transmisión sexual

En el caso de los hombres se les echa cerveza o limón en el pene y ya se da cuenta uno, con el brinco de ellos.

Si yo voy a tener relaciones sexuales, siempre voy a usar condón.

La forma de prevenir las convulsiones es mediante el control en el consumo de sustancias psicoactivas.

A mi casi no me daban ataques, yo era por lo mismo menos consumo de drogas.

Los niños en situación de calle afirman que una forma de prevenir las enfermedades es manejar los afectos y los sentimientos, porque el estrés que genera la vida en la calle facilita y agrava las enfermedades.

A veces caigo en el error de tensionarme mucho estresarme mucho. Son situaciones que generan mucha angustia, mucha impotencia. Estoy tratando de manejar mis afectos, mis sentimientos

En el caso de las heridas la prevención de infección se realiza aislando el contacto con aquellos que los pueden contaminar y que no pertenecen al mundo de personas en situación de calle y si le van a curar la herida exigen que la persona que les va a realizar la curación, se lave muy bien las manos, por ningún motivo 
aceptan que les laven o desinfecten las heridas, pues piensan que estas se pueden infectar. En la práctica esto ha podido ser comprobado por los investigadores al tratarles las heridas y se ha encontrado que cuando estas no se lavan, no solamente no se infectan, sino que cicatrizan más rápidamente.

\section{Discusión}

Los criterios de clasificación que tienen los niños en situación de calle en relación con las enfermedades, las heridas y el tratamiento, no coinciden con los criterios de la epidemiologia clásica.

Para autores como Orozco (2020), las condiciones precarias en que viven los niños en situación de calle les producen enfermedades tales como venéreas, tuberculosis, sida y hepatitis, sin embargo, en este estudio los niños, no relatan sufrir enfermedades como tuberculosis y hepatitis. Orozco (2020), plantea, al igual que lo encontrado en este trabajo, que las causas de las heridas están relacionadas con las interacciones entre ellos mismos, otros habitantes de calle y la fuerza pública, lo mismo plantea Monsalve, Arcila, Suescún y Cardona (2016), pero le agrega como generadora de heridas, la accidentalidad, principalmente por los vehículos automotores.

Concordamos con Forero, Giraldo, Valencia, Hurtado y Montoya (2007), en lo que se refiere al temor que los niños relatan frente al sida, porque genera rechazo de los otros y con Morais (2005), quien plantea que en su estudio sobre niños en situación de calle halló que estos manifiestan miedo al sida, por ser una enfermedad que no tiene cura y que mata, aunque el sida no es una enfermedad común en esta población, dado que toman medidas para evitarlo.

Según Sontag (2003), las enfermedades como el sida y el cáncer son consideradas misteriosas y generan prejuicios, fobias y miedos. Coincidimos con la autora, ya que los niños manifiestan sentir miedo de contraer sida, porque les genera rechazo de sus compañeros y los lleva a la muerte.

Goffman (2006) plantea que el término estigma se refiere al mal en sí mismo y no a las manifestaciones corporales pues es más bien "un atributo profundamente desacreditador" (p.13). Los niños en situación de calle, al adquirir el sida son estigmatizados por las connotaciones que tiene la enfermedad: no tener curación ser mortal y ser fácilmente transmisible. Lo mismo ocurre cuando asisten a las instituciones de salud en las que los malos tratos y el señalamiento no se refieren tanto al problema de salud que tienen en ese momento, como por su condición de niños en situación de calle y les ponen obstáculos para ser atendidos. Al respecto, Morais (2005) afirma: "los moradores de calle son discriminados en los hospitales y si no tienen documentos es difícil que puedan ingresar"

Coincidimos con Abadíe (2009) en que los niños se adaptan a las condiciones de la calle y por esta razón tienen más alto el umbral del dolor y no adquieren las enfermedades propias de los niños y aunque el manejo de sus heridas no es el convencional cicatrizan fácilmente y no se infectan. La mugre denominada por los niños "el mugre" representa un factor de protección que evita las infecciones; en el estudio de Monsalve-Escudero et al. (2016) esta mugre debe permanecer en la piel, pero no puede entrar en la herida porque se produce la infección. Lo encontrado en este estudio difiere en cuanto a que la mugre representa una barrera de protección y no importa si está en la piel o dentro de la herida.

Lo hallado en esta investigación en relación con la clasificación y el tratamiento de las enfermedades y las heridas se relaciona con lo planteado por Menéndez (1994), quien considera que una de las maneras de resolver los padecimientos físicos y emocionales es mediante la autoatención que se realiza según la cultura y las alternativas disponibles.,

Según Oliveira (2011), el autocuidado se relaciona con la capacidad que tiene el individuo de definir su propia conducta y se da con la interacción y los determinantes socioculturales. Esto mismo sucede con lo encontrado en este estudio, pues los niños al considerarse libres actúan de forma autónoma y por lo tanto toman las decisiones sobre sus enfermedades, de lo que han aprendido en las interacciones que establecen en la calle.

El concepto del hospital como el sitio para morir coincide con lo planteado por Ariés (1984), para quien este está reservado a los enfermos graves y es el lugar de la muerte normal.

Los resultados de este estudio coinciden con Monsalve-Escudero et al. (2016), quienes plantea 
que los niños evitan hacer uso del hospital por considerarlo un lugar donde son rechazados y estigmatizados, tanto por el personal de salud como por los otros pacientes, además las medidas de aseo que se toman en los hospitales para la atención de las heridas son lesivas, ya que aumentan los riesgos de infección.

\section{Conclusiones}

Los niños definen alteraciones en la salud a las enfermedades y heridas.

Los niños clasifican las alteraciones en la salud como graves, que son las que llevan a la muerte; serias las que coartan la libertad y leves las que permiten continuar con su vida cotidiana.

Hay alteraciones en la salud causadas por Dios y por el hombre, las primeras no son prevenibles y las causadas por el hombre si son prevenibles.

Las alteraciones graves deben ser tratadas en el hospital y generalmente terminan en muertes.

Las alteraciones serias coartan la libertad y deben buscar a personas que les ayuden.

Las alteraciones leves no coartan la libertad, se tratan con autocuidado.

Una condición fundamental para que sus heridas no se infecten es no realizar ningún procedimiento antiséptico en ellos, pero sí en las personas que los van $a$ atender.

La estigmatización, especialmente en las instituciones de salud (hospitales) es muy fuerte con esta población.

Las alteraciones en la salud para los niños en situación de calle significan experiencia.

Las heridas para los niños en situación de calle significan venganza, valentía o vergüenza.

\section{Recomendaciones}

Las recomendaciones de este trabajo están sustentadas en dos miradas: las de los niños en situación de calle y las de los investigadores.

Para los niños en situación de calle es fundamental que no solo en los servicios de salud, sino en su vida diaria, las personas entiendan que ellos son seres humanos y que por lo tanto no deben ser discriminados, ni rechazados. En el caso de las instituciones de salud, los niños proponen que el personal de salud sea formado para que los entiendan y para ello proponen que los estudiantes de las distintas áreas de la salud sean llevados a las calles, para que interactúen con las personas que las habitan y conozcan su situación.

Desde la mirada de los investigadores, se sugiere que las personas del área de la salud y de los investigadores, en general, realicen investigaciones, con el fin de conocer los tipos de enfermedades y heridas, y las distintas formas de manejo que les dan los niños, para que una vez los reciban en las consultas entiendan, no solo la forma como las conciben, sino las formas como las manejan.

Diseñar propuestas encaminadas a apoyar a los niños en situación de calle, en prevenir las enfermedades relacionadas en este articulo y en el cuidado de las heridas producidas por vivir en la calle. Además fortalecer la atención integral, sicosocial encaminada a la promoción de la salud.

\section{Agradecimientos}

\begin{abstract}
A los niños, niñasy adolescentes en situación de calle, especialmente a quienes participaron en el Estudio. A la Universidad de Antioquia y a las Facultades de Salud Pública y Enfermería por facilitarnos el tiempo para llevar a cabo la investigación.
\end{abstract}

\section{Contribuciones de los autores}

Todos los autores contribuyeron en todas las etapas de la investigación y redactaron el informe de investigación.

\section{Conflictos de interés}

No se han declarado conflictos financieros, legales o políticos que involucren a terceros (gobierno, empresas y fundaciones privadas, 
etc.) para ningún aspecto del trabajo presentado (incluidas, entre otras, subvenciones y financiación, participación en el consejo asesor, diseño del estudio, preparación del manuscrito, análisis estadístico, etc.).

\section{Referencias}

Abadíe, M. (2009). Condiciones de salud, percepciones y significados del proceso salud/enfermedad/atención de los niños/niñas que trabajan, de 8 a 14 años, un estudio de caso, en el barrio IAPI de Quilmes en el año 2009 [Tesis de maestría, Cedes-Flacso]. Repositoria Flacso Andes. http://hdl.handle. net/10469/3400

Akman, M., Arousin, J., Pignata, N., Pojomovsky, J., Riterman, F., Triñares, G., \& Waisman, M. (1999). Modalidades de abordaje médico a chicos de la calle en hospitales públicos de la Ciudad de Buenos Aires. Archivos argentinos de pediatría, 97(4), 241-248. https://www.sap.org.ar/docs/ publicaciones/archivosarg/1999/99 241 248.pdf

Ariès, P. (1984). El Hombre Ante La Muerte. Taurus. https://www. academia.edu/7327574/El hombre ante la muerte Philippe_Aries_Libro

Aristizábal Monsalve, T. M., Giraldo Pineda, Á., \& Forero Pulido, C. (2020). Significado de las heridas y su cuidado en niños niñas y adolescentes en situación de calle. New Trends in Qualitative Research, 4, 14-25. https://doi.org/10.36367/ ntqr.4.2020.14-25

Castillo, E., \& Vásquez, M. L. (2003). El rigor metodológico en la investigación cualitativa. Colombia Médica, 34(3), 164-167. https://www.redalyc.org/pdf/283/28334309.pdf

Decreto Ley 356 de 1994 [República de Colombia, Ministerio de Defensa Nacional]. Por el cual se expide el Estatuto de Vigilancia y Seguridad Privada. Diario Oficial No 41.220, de 11 de febrero de 1994. https://www.funcionpublica.gov. co/eva/gestornormativo/norma.php?i=1341

Forero Pulido, C., Giraldo Pineda, Á., Valencia González, A., Hurtado Gutiérrez, M. A., \& Montoya Giraldo, B. (2007). Para sobrevivir en la calle hay que tener miedo. Investigación y Educación en Enfermería, 25(2), 28-35. https://www.redalyc.org/articulo.oa?id=105215257002

Forselledo, A. G. (2001). Niñez en situación de calle, un modelo de prevención de las farmacodependencias basado en los derechos humanos. Boletín del Instituto Interamericano del Niño, 236, 49-80. http://iin.oea.org/Cursos_a_distancia/ Ninez_en_situacion_de_calle.pdf

Geertz, C. (2003). La interpretación de las culturas. Gedisa.
Goffman, E. (2006). Estigma: La identidad deteriorada. Amorrortu.

Instituto Colombiano de Bienestar Familiar. (2014). Análisis de la situación de vida en calle de niños, niñas y adolescentes en Colombia. Observatorio del Bienestar de la Niñez. https:// www.icbf.gov.co/programas-y-estrategias/observatorio-delbienestar-de-la-ninez/analisis-de-la-situacion-de-vida-en

Menéndez, E. (1994). La enfermedad y la curación, ¿Qué es medicina tradicional? Alteridades, 4(7), 71-83. https://www. redalyc.org/pdf/747/747111357008.pdf

Monsalve-Escudero, L. M., Arcila-García, V. J., Suescun-Durango, B. A., \& Cardona- Arias, J. A. (2016). Representaciones sociales sobre salud en adolescentes habitantes de calle, Medellín 2014. Médicas UIS, 29(3), 33-42. http://www.scielo. org.co/pdf/muis/v29n3/0121-0319-muis-29-03-00033.pdf

Morais, N. A. (2005). Um estudo sobre a saúde de adolescentes em situação de rua: o ponto de vista de adolescentes, profissionais de saúde e educadores [Un estudio sobre la salud de los adolescentes en situación de calle: el punto de vista de los adolescentes, los profesionales de la salud y los educadores] [Tesis de maestría, Universidade Federal do Rio Grande do Sul]. Lume. http://hdl.handle. net/10183/7392

Oliveira, D. L. L. C. (2011). Enfermagem e suas apostas no autocuidado: investimentos emancipatórios ou práticas de sujeição? [La enfermería y sus apuestas en el autocuidado: ¿inversiones emancipatórias o prácticas de sujeción?]. Revista Brasileira de Enfermagem, 64, 185-188. https://doi. org/10.1590/S0034-71672011000100027

Orozco Salazar, E. B. (2020). Habitantes en situación de calle y construcción territorial en el centro Occidente de Medellín. Revista de la Facultad de Trabajo Social, 23(23), 137-147. https://revistas.upb.edu.co/index.php/trabajosocial/ article/view/2727

Pasamonik, G. (2009). Salud, infancia y adolescencia: prácticas de atención de niños, niñas y adolescentes en situación de calle en la ciudad de Buenos Aires. Anuario de Investigaciones, 16, 257-266. https://www.redalyc.org/ pdf/3691/369139945023.pdf

Resolución 008430 de 1993 [Ministerio de Salud de Colombia]. Por la cual se establecen las normas científicas, técnicas y administrativas para la investigación en salud. 4 de octubre de 1993. https://www.hospitalsanpedro.org/ images/Comite_Investigacion/Resolucion_8430_de_1993. pdf

Sontag, S. (2003). La enfermedad y sus metáforas El Sida y sus metáforas. Santillana ediciones.

United Nations Children's Emergency Fund. (1989). Convención sobre el derecho de los niños. https://www.unicef.org/es/ convencion-derechos-nino/convencion-version-ninos 